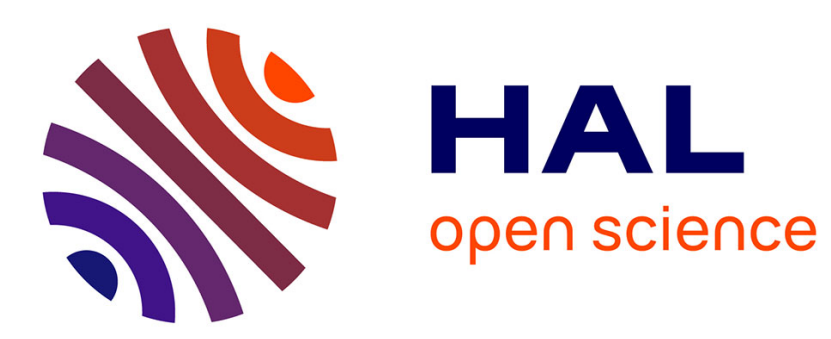

\title{
Attraction domain estimation of linear controllers for the attitude control of VTOL vehicles: P/PI control of a quadrotor
}

Jeremy Barra, Gérard Scorletti, Suzanne Lesecq, Mykhailo Zarudniev, Eric Blanco

\section{To cite this version:}

Jeremy Barra, Gérard Scorletti, Suzanne Lesecq, Mykhailo Zarudniev, Eric Blanco. Attraction domain estimation of linear controllers for the attitude control of VTOL vehicles: P/PI control of a quadrotor. ECC, May 2020, Saint Petersburg, Russia. 10.23919/ECC51009.2020.9143612 . hal-03230678

\section{HAL Id: hal-03230678 https://hal.science/hal-03230678}

Submitted on 20 May 2021

HAL is a multi-disciplinary open access archive for the deposit and dissemination of scientific research documents, whether they are published or not. The documents may come from teaching and research institutions in France or abroad, or from public or private research centers.
L'archive ouverte pluridisciplinaire HAL, est destinée au dépôt et à la diffusion de documents scientifiques de niveau recherche, publiés ou non, émanant des établissements d'enseignement et de recherche français ou étrangers, des laboratoires publics ou privés. 


\title{
Attraction domain estimation of linear controllers for the attitude control of VTOL vehicles: P/PI control of a quadrotor
}

\author{
Jeremy Barra ${ }^{1,3}$, Gerard Scorletti ${ }^{3}$, Suzanne Lesecq $^{1}$, Mykhailo Zarudniev $^{2}$, Eric Blanco ${ }^{3}$
}

\begin{abstract}
In this paper, we present a numerical method based the Lyapunov theory to estimate the attraction domain of a class of nonlinear systems. This problem is motivated by the analysis of linear attitude controllers for the control of Vertical Take-Off and Landing (VTOL) vehicles such as quadrotors. These linear controllers are typically designed in order to ensure local stability around the hover point. The purpose of this work is to estimate their attraction domain around this point. The proposed attraction domain estimation method requires to solve a convex optimization problem involving parameter-dependent Linear Matrix Inequalities (LMI). This problem is generally difficult to solve as it is an infinite dimensional optimization problem. However, we reveal that the specific structure of the VTOL vehicles attitude model can be exploited to make this LMI problem finite dimensional and thus numerically solvable.
\end{abstract}

\section{INTRODUCTION}

Unmanned Aerial Vehicles (UAVs) have gained significant attention in the recent years. Depending on the application domain, the UAV may vary in shape, size and weight. Here, we focus on Vertical Take-Off and Landing (VTOL) vehicles, especially on quadrotors. The following work is based on the experimental quadrotor platform Bitcraze Crazyflie 2.0 which was used to validate the nonlinear model under study.

The feedback control of such vehicles has been widely addressed in the literature, see e.g. [1], [2]. The literature shows that the complex dynamics of the quadrotors represent a challenge from a control point-of-view. Indeed, the quadrotor model exhibits nonlinear dynamics, coupling between control axes and uncertainties such as aerodynamical effects [3], [4]. To address the control problem, the literature reports two main approaches, namely, nonlinear and linear control design techniques. The nonlinear design techniques account for these complex dynamics during the design process and ensure the stability for the nonlinear model on a large operating domain [5], [6]. However, they may lead to a complex control law design and implementation process. The linear control design techniques rely on a linearised model of the quadrotor, generally at the hover point, which simplifies the dynamics because the axes are decoupled at this equilibrium point. Classical controllers can then be used, such as off-the-shelf Proportional-Integral-Derivative (PID) controllers designed for each axes [7]. The main drawback of the linear control design techniques is that they do not theoretically guarantee the stability of the quadrotor far from the linearisation point. In this paper, we use these linear control design techniques for the control of the experimental

\footnotetext{
${ }^{1}$ Université Grenoble Alpes, CEA-LIST, Grenoble, France, ${ }^{2}$ Université Grenoble Alpes, CEA-LETI, Grenoble, France, ${ }^{3}$ Laboratoire Ampère CNRS, Université de Lyon, École Centrale de Lyon, Écully Cedex, France
}

quadrotor platform Bitcraze Crazyflie 2.0. We experimentally observe stable flight of the quadrotor even far from the linearisation point where couplings have a non-negligible effect. Nevertheless, a formal proof on the model of this nice behaviour could improve the design process of the linear controllers.

The contribution of the present work is to give a numerical method based on the Lyapunov theory to estimate the attraction domain for a class of nonlinear control systems that includes those represented by the VTOL attitude model. This method relies on a Linear Matrix Inequalities (LMI) problem formulation to parametrise the search for a Lyapunov function as a convex optimization problem.

This proposed method is applied to the analysis of the linear attitude control of a quadrotor. First, P/PI controllers are synthesised for a linearisation of the quadrotor attitude model at the hover point using standard linear control design techniques. Then, our numerical method is used to estimate the attraction domain of these controllers around the hover point when they are used to control the quadrotor nonlinear dynamics. The parametrisation of this problem by the attitude angles of the UAV leads to a parameter-dependent LMI of infinite dimension which is not solvable numerically [8]. Consequently, we propose a solution that exploits the specific structure of the VTOL attitude model to make this LMI problem finite dimensional and thus solvable numerically.

The paper is organised as follows. For the sake of completeness, we first present in section II the well-known quadrotor dynamical model and in section III the design of the linear attitude controllers. Then, in section IV, we develop our contribution, which is a method based on a LMI problem formulation to estimate the attraction domain for a class of nonlinear control systems that includes the VTOL attitude model. This method is then applied to the attraction domain estimation of the quadrotor attitude controllers previously designed in section III.

\section{QUADROTOR ROTATIONAL DYNAMICS}

In this section, we present the equations of the rotational motion of the quadrotor. Then, the attitude nonlinear model is linearised about an equilibrium point to gain insight about its structure and stability.

\section{A. Equations of motion}

Consider the body-fixed referential $\mathcal{R}_{b}=\left(G, \vec{x}_{b}, \vec{y}_{b}, \vec{z}_{b}\right)$ and the inertial one $\mathcal{R}_{i}=\left(O_{i}, \vec{x}_{i}, \vec{y}_{i}, \vec{z}_{i}\right)$, see figure 1 . The equations of motion are given under usual assumptions:

- the quadrotor is a rigid-body of constant mass; 
- its structure is symmetrical about the $\vec{x}_{b}$ and $\vec{y}_{b}$ axes;

- the origin $G$ of $\mathcal{R}_{b}$ is the quadrotor center of gravity.

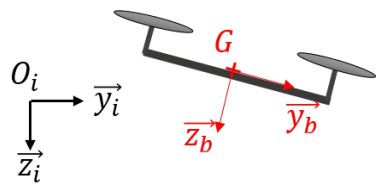

Fig. 1: Body-fixed and inertial referentials

The attitude state vector is given by $x=\left[\omega^{\top}, \eta^{\top}\right]^{\top}$ where $\omega=\left[\omega_{x}, \omega_{y}, \omega_{z}\right]^{\top}$ are the angular velocities expressed in $\mathcal{R}_{b}$ and $\eta=[\phi, \theta, \psi]^{\top}$ (resp. roll, pitch, yaw) are the quadrotor attitude Euler angles expressed in $\mathcal{R}_{i}$. The attitude input vector is $u=\left[\tau_{x}, \tau_{y}, \tau_{z}\right]^{\top}$ where $\tau=\left(\tau_{x}, \tau_{y}, \tau_{z}\right)$ are the moments created by the rotors. From a standard NewtonEuler modelling formalism [9], we get:

$$
\left\{\begin{array}{l}
\dot{\omega}_{x}=\frac{1}{J_{x}}\left[\tau_{x}-\left(J_{z}-J_{y}\right) \omega_{y} \omega_{z}\right] \\
\dot{\omega}_{y}=\frac{1}{J_{y}}\left[\tau_{y}-\left(J_{x}-J_{z}\right) \omega_{x} \omega_{z}\right] \\
\dot{\omega}_{z}=\frac{1}{J_{z}}\left[\tau_{z}-\left(J_{y}-J_{x}\right) \omega_{x} \omega_{y}\right] \\
\dot{\phi}=\omega_{x}+s_{\phi} t_{\theta} \omega_{y}+c_{\phi} t_{\theta} \omega_{z} \\
\dot{\theta}=c_{\phi} \omega_{y}-s_{\phi} \omega_{z} \\
\dot{\psi}=\frac{s_{\phi}}{c_{\theta}} \omega_{y}+\frac{c_{\phi}}{c_{\theta}} \omega_{z}
\end{array}\right.
$$

where $c_{i}=\cos (i), s_{i}=\sin (i), t_{i}=\tan (i) . J$ is the quadrotor inertia matrix such that:

$$
J=\operatorname{diag}\left(J_{x}, J_{y}, J_{z}\right)=\left[\begin{array}{ccc}
J_{x} & 0 & 0 \\
0 & J_{y} & 0 \\
0 & 0 & J_{z}
\end{array}\right]
$$

\section{B. Model linearisation}

The nonlinear model (1) is linearised in order to apply classical linear control theory tools. Usually, the quadrotor model is linearised at the hover equilibrium point $\eta=$ $[0,0,0]^{\top}[9]$. Here, we linearise the model about a general equilibrium point $\bar{x}=[0,0,0, \bar{\phi}, \bar{\theta}, \bar{\psi}]^{\top}$ :

$$
\left\{\begin{array}{l}
\delta \dot{\omega}_{x}=\frac{\delta \tau_{x}}{J_{x}} \\
\delta \dot{\omega}_{y}=\frac{\delta \tau_{y}}{J_{y}} \\
\delta \dot{\omega}_{z}=\frac{\delta \tau_{z}}{J_{z}} \\
\delta \dot{\phi}=\delta \omega_{x}+s_{\bar{\phi}} t_{\bar{\theta}} \delta \omega_{y}+c_{\bar{\phi}} t_{\bar{\theta}} \delta \omega_{z} \\
\delta \dot{\theta}=c_{\bar{\phi}} \delta \omega_{y}-s_{\bar{\phi}} \delta \omega_{z} \\
\delta \dot{\psi}=\frac{s_{\bar{\phi}}}{c_{\bar{\theta}}} \delta \omega_{y}+\frac{c_{\bar{\phi}}}{c_{\bar{\theta}}} \delta \omega_{z}
\end{array}\right.
$$

The linear model (3) exhibits a cascade structure with 2 subsystems related to the angular velocities and positions. The model can be represented as a set of gains and integrators with couplings between axes parametrised by the attitude angles of the quadrotor. We adopt for the controllers design the common assumption that the quadrotor is close to the hover point [9] [10]. In that case, the couplings vanish, allowing to use a decoupled control law on each axis.

\section{DESIGN OF ATTITUDE CONTROLLERS}

We now present the design of the attitude controllers of the quadrotor. Several linear and nonlinear control designs have already been proposed in the literature for such vehicles. We focus here on linear control design methods as we aim to implement classical PID controllers. The controllers outputs feed the rotors to modify their rotating speeds and generate torques that modify the attitude of the quadrotor. For the sake of simplicity, we consider hereafter that the relation between the control inputs and the physical torques is given by a constant gain $D=\operatorname{diag}\left(D_{x}, D_{y}, D_{z}\right)$, see figure 2 .

\section{A. P/PI control design}

For each axis, the control of the attitude is performed using 2 nested control loops exploiting the cascade structure of (3). The nested controllers respectively track the angular velocity and the attitude angle thanks to the structure presented on figure 2 for the $\vec{x}_{b}$ axis as an example. The chosen controllers are given by:

$$
K_{\omega}=k_{p_{\omega}} \quad, \quad K_{\eta}=k_{p_{\eta}}+\frac{k_{i_{\eta}}}{s}
$$

where $k_{p_{\omega}}=\operatorname{diag}\left(k_{p, \omega_{x}}, k_{p, \omega_{y}}, k_{p, \omega_{z}}\right), \quad k_{p_{\eta}}=$ $\operatorname{diag}\left(k_{p, \phi}, k_{p, \theta}, k_{p, \psi}\right)$ and $k_{i_{\eta}}=\operatorname{diag}\left(k_{i, \phi}, k_{i, \theta}, k_{i, \psi}\right)$.

The internal loop dealing with the angular velocities should be tuned to be as fast as possible to not interfere with the external loop related to the attitude angles. This is realised by an inner proportional controller $K_{\omega}$, whose diagonal gains are chosen large enough so that the bandwidth of the inner loop is much higher than the one of the outer loop. The outer controller $K_{\eta}$ contains proportional gains and an integral action is introduced to account for unmodelled dynamics and unknown disturbances such as wind gusts. Considering that the inner feedback can be reduced from the external loop perspective to a gain of 1 , the gains of $K_{\eta}$ are then tuned to maximize the bandwidth of the system while guaranteeing sufficient phase and gain margins.

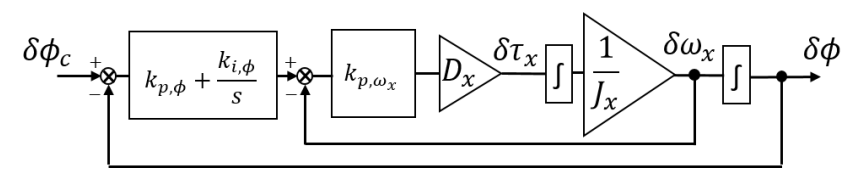

Fig. 2: Control structure of the roll angle with 2 nested loops

Figure 3 presents the step response of the attitude system when the P/PI control law is applied both on the linear and on the nonlinear models of our experimental quadrotor platform Bitcraze Crazyflie 2.0. Both systems are stable, but their dynamics differ significantly because of the couplings between the axes on the nonlinear system. These couplings have a non-negligible effect even for relatively low attitude angles of $20 \mathrm{deg}$. Moreover, as it is developed for the linearised model, the stability of the quadrotor coupled with the P/PI control law is, at this point, only guaranteed close to the hover position. Therefore, it appears necessary to perform a deeper analysis of this controlled system to ensure that instability does not occur during a flight condition different from the hover point.

\section{STABiLity ANALYSIS}

We first present in this section a general stability theorem for a given class of nonlinear systems. Then, we show that 

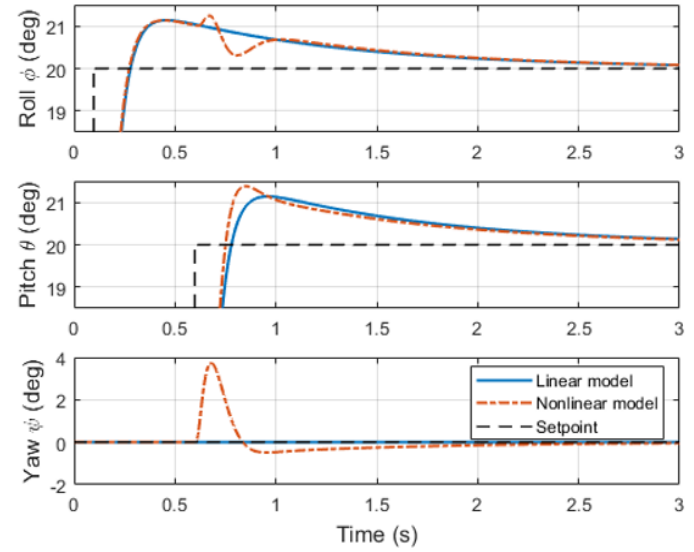

Fig. 3: Step responses of the linear and the nonlinear systems

the VTOL attitude model belongs to this class of nonlinear systems and we adapt more specifically the theorem to the attraction domain estimation for this case. Finally, the analysis is applied numerically to the attraction domain estimation of P/PI attitude controllers designed for our experimental quadrotor platform Bitcraze Crazyflie 2.0.

\section{A. Motivation}

Stability analysis of a closed-loop system is typically performed using the Lyapunov theory. Classically, one chooses an ad-hoc Lyapunov function given the system model and the control law. This choice may be complex, and the control law sometimes requires additional terms to simplify the equations hence the stability proof. Such an approach is for example adopted in [11], [6] where PID-like control laws containing additional cancelling terms are proven to stabilize the attitude of a quadrotor. In this paper, we consider the search for a quadratic Lyapunov function as a convex optimization problem involving Linear Matrix Inequalities (LMI) that can be solved numerically [12]. Compared to the classical approach, the LMI approach leads to a more systematic method for proving the system stability.

\section{B. General stability theorem}

Consider a nonlinear system given in the form:

$$
\dot{x}=\left(A(\Delta(x))+A_{s k}(x)\right) x
$$

with

- $A(\Delta(x))=\Delta(x) \star M$ with $\Delta(x)$ a block-diagonal matrix, $M=\left[\begin{array}{l|l}M_{11} & M_{12} \\ \hline M_{21} & M_{22}\end{array}\right]$ and $\star$ the Redheffer star product defined here by $\Delta(x) \star M=M_{22}+$ $M_{21} \Delta(x)\left(I-M_{11} \Delta(x)\right)^{-1} M_{12}$ where $I$ is the identity matrix.

- $A_{s k}(x)$ is a skew-symmetric matrix such that $A_{s k}(x)=$ $\sum_{i=1}^{n} x_{i} A_{s k, i}, A_{s k, i}=-A_{s k, i}^{\top}$

We want to evaluate the stability of the equilibrium point $x=0$ which is the origin of the system (5). In the following theorem, we give the conditions under which this equilibrium point $x=0$ is globally asymptotically stable.
Theorem 1. For the system (5), if the matrices $P=P^{\top}>0$ and $W=W^{\top}$ exist such that the following statements hold true:

$$
\begin{gathered}
{\left[\begin{array}{c}
I \\
\Delta(x)
\end{array}\right]^{\top} W\left[\begin{array}{c}
I \\
\Delta(x)
\end{array}\right] \geq 0, \forall x \in \mathbb{R}^{n}} \\
{\left[\begin{array}{cc}
M_{11} & M_{12} \\
I & 0 \\
M_{21} & M_{22} \\
0 & I
\end{array}\right]^{\top}\left[\begin{array}{cc}
W & 0 \\
0 & {\left[\begin{array}{cc}
0 & P \\
P & 0
\end{array}\right]}
\end{array}\right]\left[\begin{array}{cc}
M_{11} & M_{12} \\
I & 0 \\
M_{21} & M_{22} \\
0 & I
\end{array}\right]<0} \\
A_{s k, i}^{\top} P+P A_{s k, i}=0, i=1, \ldots, n
\end{gathered}
$$

then the origin $x=0$ of the system (5) is globally asymptotically stable.

Proof. Consider the equation $y=(\Delta(x) \star M) x$, associated with a linear-fractional representation (LFR) given on figure 4 that corresponds to the following equations:

$$
\begin{gathered}
p=\Delta(x) q \\
\left\{\begin{array}{l}
q=M_{11} p+M_{12} x \\
y=M_{21} p+M_{22} x
\end{array}\right.
\end{gathered}
$$

where $p$ and $q$ are internal signals of the system.

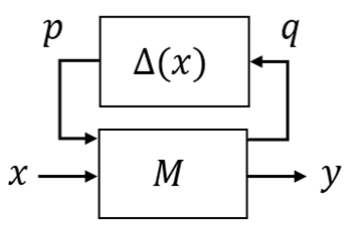

Fig. 4: Linear fractional representation corresponding to (9)

From equation (7), given the matrices $P=P^{\top}>0$ and $W=W^{\top}$ satisfying the theorem 1 , we can write the following quadratic form [8]:

$\left[\begin{array}{l}p \\ x\end{array}\right]^{\top}\left[\begin{array}{cc}M_{11} & M_{12} \\ I & 0 \\ M_{21} & M_{22} \\ 0 & I\end{array}\right]^{\top}\left[\begin{array}{cc}W & 0 \\ 0 & {\left[\begin{array}{ll}0 & P \\ P & 0\end{array}\right]}\end{array}\right]\left[\begin{array}{cc}M_{11} & M_{12} \\ I & 0 \\ M_{21} & M_{22} \\ 0 & I\end{array}\right]\left[\begin{array}{l}p \\ x\end{array}\right]<0$

which according to equation (9b) gives:

$$
\left[\begin{array}{l}
q \\
p
\end{array}\right]^{\top} W\left[\begin{array}{l}
q \\
p
\end{array}\right]+y^{\top} P x+x^{\top} P y<0
$$

From equation (6) and (9a), we have:

$$
q^{\top}\left[\begin{array}{c}
I \\
\Delta(x)
\end{array}\right]^{\top} W\left[\begin{array}{c}
I \\
\Delta(x)
\end{array}\right] q \geq 0 \Leftrightarrow\left[\begin{array}{l}
q \\
p
\end{array}\right]^{\top} W\left[\begin{array}{l}
q \\
p
\end{array}\right] \geq 0
$$

which gives from equation (11):

$$
y^{\top} P x+x^{\top} P y<0
$$

From equation (8), we have [13]:

$\sum_{i=1}^{n} x_{i}^{\top} A_{s k, i}^{\top} P+P A_{s k, i} x_{i}=0 \Leftrightarrow A_{s k}(x)^{\top} P+P A_{s k}(x)=0$

leading to:

$$
x^{\top}\left(A_{s k}(x)^{\top} P+P A_{s k}(x)\right) x=0
$$


Now, from (13) and (15), we get:

$$
\dot{x}^{\top} P x+x^{\top} P \dot{x}<0
$$

that is, $\dot{V}(x)<0$ for $x$ solution of (5) and with $V(x)=$ $x^{\top} P x>0, x \neq 0$ which is a Lyapunov function. We therefore conclude that the origin of the system (5) is asymptotically stable.

To guarantee the stability of the system (5), a matrix $W=$ $W^{\top}$ should be determined such that (6) holds true. Such a matrix cannot be found generally as it involves a parameterdependent LMI which requires to solve an infinite number of LMI. However, a matrix $W$ can be found when $\Delta(x)$ exhibits a specific structure, which is the case hereafter.

\section{VTOL attraction domain}

The previous theorem is now applied to the attraction domain estimation of linear VTOL attitude controllers. Consider the attitude system given in (1) which is common for most VTOL vehicles such as the quadrotor. It is written in the compact form hereafter :

$$
\left\{\begin{array}{l}
J \dot{\omega}=-\hat{\omega} J \omega+u \\
\dot{\eta}=S(\eta)^{-1} \omega
\end{array}\right.
$$

with $u=D\left(-k_{p_{\omega}} \omega-k_{p_{\eta}} \eta-k_{i_{\eta}} x_{i}\right)$ where $\dot{x}_{i}=\eta$, $D=\operatorname{diag}\left(D_{x}, D_{y}, D_{z}\right)$ is the diagonal matrix of gains corresponding to the relation between the outputs of the controllers and the physical torques and:

$\hat{\omega}=\left[\begin{array}{ccc}0 & -\omega_{z} & \omega_{y} \\ \omega_{z} & 0 & -\omega_{x} \\ -\omega_{y} & \omega_{x} & 0\end{array}\right], S(\eta)^{-1}=\left[\begin{array}{ccc}1 & s_{\phi} t_{\theta} & c_{\phi} t_{\theta} \\ 0 & c_{\phi} & -s_{\phi} \\ 0 & s_{\phi} / c_{\theta} & c_{\phi} / c_{\theta}\end{array}\right]$

This attitude system can be written under the form (5) where the skew-symmetric part is given by $\hat{\omega}$. Indeed, consider the change of variable $z=J \omega$ and the state vector $x=$ $\left[z^{\top}, \eta^{\top}, x_{i}^{\top}\right]^{\top}$, the system (17) can be written as:

$$
\left[\begin{array}{c}
\dot{z} \\
\dot{\eta} \\
\dot{x_{i}}
\end{array}\right]=(\underbrace{\left[\begin{array}{ccc}
-D k_{p_{\omega}} J^{-1} & -D k_{p_{\eta}} & -D k_{i_{\eta}} \\
S(\eta)^{-1} J^{-1} & 0 & 0 \\
0 & I & 0
\end{array}\right]}_{A(\Delta(x))}+\underbrace{\left[\begin{array}{ccc}
-\hat{\omega} & \cdots & 0 \\
\vdots & \ddots & \vdots \\
0 & \cdots & 0
\end{array}\right]}_{A_{s k}(x)})\left[\begin{array}{c}
z \\
\eta \\
x_{i}
\end{array}\right]
$$

The time-varying matrix $S(\eta)^{-1}$ can be represented in the LFR form as an interconnection between a term $\Delta(x)$ depending on the value of the attitude angles $\eta$ and a static system $G=\left[\begin{array}{l|l}G_{11} & G_{12} \\ \hline G_{21} & G_{22}\end{array}\right]$. As a consequence, the full $A(\Delta(x))$ matrix admits a LFR representation $A(\Delta(x))=$ $\Delta(x) \star M=$ where:

$$
\begin{aligned}
& M_{11}=G_{11} \quad M_{12}=\left[G_{12} J^{-1} \quad 0 \quad 0\right] \\
& M_{21}=\left[\begin{array}{c}
0 \\
G_{21} \\
0
\end{array}\right] \quad M_{22}=\left[\begin{array}{ccc}
-D k_{p_{\omega}} J^{-1} & -D k_{p_{\eta}} & -D k_{i_{\eta}} \\
G_{22} J^{-1} & 0 & 0 \\
0 & I & 0
\end{array}\right]
\end{aligned}
$$

and where the $G_{i j}$ components are given by:

$$
\begin{aligned}
G_{11} & =\left[\begin{array}{cccccc}
-1 & 0 & -1 & 0 & 0 & 0 \\
0 & -1 & 0 & -1 & 0 & -1 \\
0 & 0 & 0 & 0 & 0 & 0 \\
0 & 0 & 0 & 0 & 0 & 1 \\
0 & 0 & 0 & 0 & 0 & 0 \\
0 & -1 & 0 & -1 & 0 & -1
\end{array}\right] \quad G_{12}=\left[\begin{array}{lll}
0 & 1 & 0 \\
0 & 0 & 1 \\
0 & 0 & 0 \\
0 & 0 & 0 \\
0 & 0 & 0 \\
0 & 0 & 1
\end{array}\right] \\
G_{21} & =\left[\begin{array}{cccccc}
0 & 0 & 0 & 0 & 1 & 0 \\
-1 & 0 & -1 & 0 & 0 & 0 \\
0 & -1 & 0 & -1 & 0 & -1
\end{array}\right] \quad G_{22}=I_{3}
\end{aligned}
$$

The varying term $\Delta(x)$ is a block-diagonal matrix given by:

$$
\Delta(x)=\operatorname{diag}\left(\Delta_{\phi}, \Delta_{\phi}, \Delta_{\theta}\right)
$$

with:

$$
\Delta_{i}=\left[\begin{array}{cc}
c_{i}-1 & s_{i} \\
-s_{i} & c_{i}-1
\end{array}\right], i=(\phi, \theta)
$$

To apply theorem 1, a matrix $W=W^{\top}$ should be determined such that it satisfies the inequality (6) characterizing $\Delta(x)$ for all $x \in \mathbb{R}^{n}$, leading to a global stability property of the system. However, in the case of a quadrotor flying in normal flight conditions (attitude angles in the range of $25 \mathrm{deg}$ ), such a global property could be too conservative. Thus, we introduce the following bounded set:

$$
B_{r}=\left\{x|| x_{4}\left|\leq \phi_{\max },\right| x_{5} \mid \leq \theta_{\max }\right\}
$$

with $x_{4}=\phi$ and $x_{5}=\theta$. Then, the inequality (6) of theorem 1 can be modified such that:

$$
\left[\begin{array}{c}
I \\
\Delta(x)
\end{array}\right]^{\top} W\left[\begin{array}{c}
I \\
\Delta(x)
\end{array}\right] \geq 0, \forall x \in B_{r}
$$

leading to a local stability version of theorem 1 .

Lemma 1. For the specific structure of $\Delta(x)$ given by (21) and for $\theta \in\left[-\theta_{\max }, \theta_{\max }\right]$ (resp. $\left.\phi\right)$, the inequality (24) is satisfied by any matrix $W \in \mathcal{W}$ such that:

$$
\begin{aligned}
& \mathcal{W}=\{W \text { such that: } \\
& \left.W=\left[\begin{array}{cc}
{\left[\begin{array}{cc}
\alpha_{\phi} S \otimes I_{2} & 0 \\
0 & \alpha_{\theta} t I_{2}
\end{array}\right]} & {\left[\begin{array}{cc}
U \otimes I_{2} & 0 \\
0 & w I_{2}
\end{array}\right]} \\
{\left[\begin{array}{cc}
U \otimes I_{2} & 0 \\
0 & w I_{2}
\end{array}\right]} & {\left[\begin{array}{cc}
(U-S) \otimes I_{2} & 0 \\
0 & (w-t) I_{2}
\end{array}\right]}
\end{array}\right]\right\}
\end{aligned}
$$

where $\otimes$ is the Kronecker product, $U=U^{\top}, S=S^{\top}>0$, $w \in \mathbb{R}, t \in \mathbb{R}_{+}$and $\alpha_{i}=2\left(1-c_{i_{\max }}\right), i=(\phi, \theta), i_{\max }=$ $\left(\phi_{\max }, \theta_{\max }\right)$.

Proof. First, note that the product $\Delta_{i}^{\top} \Delta_{i}$ is bounded:

$$
\Delta_{i}^{\top} \Delta_{i}=\left[\begin{array}{cc}
2\left(1-c_{i}\right) & 0 \\
0 & 2\left(1-c_{i}\right)
\end{array}\right] \leq \alpha_{i} I_{2}
$$

where $\alpha_{i}=2\left(1-c_{i_{\max }}\right), i=(\phi, \theta), i_{\max }=\left(\phi_{\max }, \theta_{\max }\right)$.

Consider the block-diagonal matrix $\tilde{\Delta}_{\phi}=\operatorname{diag}\left(\Delta_{\phi}, \Delta_{\phi}\right)$. For $S=S^{\top}$, we have the property that $S \otimes I_{2}$ commutes with $\tilde{\Delta}_{\phi}$. From this property, the equation (26) and for a matrix $S=S^{\top}>0$, we can write the following inequality:

$$
\left[\begin{array}{c}
I \\
\tilde{\Delta}_{\phi}
\end{array}\right]^{\top}\left[\begin{array}{cc}
\alpha_{\phi} S \otimes I_{2} & 0 \\
0 & -S \otimes I_{2}
\end{array}\right]\left[\begin{array}{c}
I \\
\tilde{\Delta}_{\phi}
\end{array}\right] \geq 0
$$


Moreover, the $\Delta_{i}$ block satisfies:

$$
\Delta_{i}+\Delta_{i}^{\top}+\Delta_{i}^{\top} \Delta_{i}=0
$$

Thus, given a matrix $U=U^{\top}$, combining the previous commutation property with (28), (27) becomes:

$$
\left[\begin{array}{c}
I \\
\tilde{\Delta}_{\phi}
\end{array}\right]^{\top}\left[\begin{array}{cc}
\alpha_{\phi} S \otimes I_{2} & U \otimes I_{2} \\
U \otimes I_{2} & (U-S) \otimes I_{2}
\end{array}\right]\left[\begin{array}{c}
I \\
\tilde{\Delta}_{\phi}
\end{array}\right] \geq 0
$$

Following the same method, the proof can be extended to the full $\Delta(x)$ matrix by introducing the scalars $t \in \mathbb{R}_{+}$and $w \in \mathbb{R}$ corresponding to the block $\Delta_{\theta}$ such that:

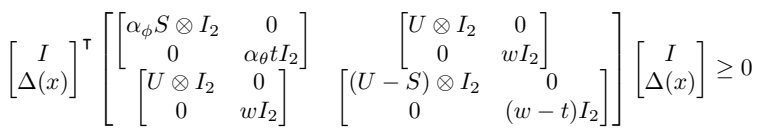

which gives the matrix $W$ stated in the lemma 1 .

The asymptotic stability property of the theorem 1 along with the inequality (24) is local as it is only defined for $x \in B_{r}$. We are now interested in estimating the domain of attraction of this local asymptotic stability property for the system (17). Consider an ellipsoid $\mathcal{E}_{p}$ defined by:

$$
\mathcal{E}_{p}=\left\{x \mid x^{\top} P x \leq 1\right\}
$$

Theorem 2. If there are matrices $P=P^{\top}>0$ and $W \in \mathcal{W}$ satisfying (7)-(8), and:

$$
\left[\begin{array}{cc}
\phi_{\max }^{2} & e_{4}^{\top} \\
e_{4} & P
\end{array}\right] \geq 0,\left[\begin{array}{cc}
\theta_{\max }^{2} & e_{5}^{\top} \\
e_{5} & P
\end{array}\right] \geq 0
$$

with $e_{i}$ is a vector of dimension $n$ where the ith element is equal to 1 and the others to 0 . Then $x=0$ is locally asymptotically stable and the ellipsoid $\mathcal{E}_{p} \subset B_{r}$ is an invariant domain of attraction.

Proof. From the proof of theorem 1, if the conditions (7)-(8) are satisfied by $P=P^{\top}>0$ and $W \in \mathcal{W}$, then $V(x)=$ $x^{\top} P x$ is a Lyapunov function such that $V(x)>0, x \neq$ 0 and $\dot{V}(x) \leq 0, \forall x \in B_{r}$ giving that $x=0$ is locally asymptotically stable. The condition (32) ensures that any initial state $x\left(t_{0}\right) \in \mathcal{E}_{p} \subset B_{r}$, see [14], [12]. It follows that as $V(x(t)) \leq V\left(x\left(t_{0}\right)\right) \leq 1, \forall t \geq 0$, the ellipsoid $\mathcal{E}_{p}$ is an invariant domain of attraction for the system (17).

The largest region $B_{r}$ can be found by manually maximizing the bounds $\theta_{\max }$ and $\phi_{\max }$. Finally, in order to find the largest ellipsoid $\mathcal{E}_{p}$ that can be contained in $B_{r}$, the following minimization problem can be solved [12]:

$$
\begin{array}{ll}
\min _{P, W \in \mathcal{W}} & \operatorname{Tr} P \\
\text { subject to } & \text { eq.(7), eq.(8), eq.(32) }
\end{array}
$$

In order to solve numerically the problem (33), the Linear Matrix Equality constraint (8) has to be eliminated. This is done by parametrizing the $P$ matrix decision variables according to (8), which can be done numerically using the Jacobi or Gauss-Seidel iteratives methods [15]. Then, the minimization problem (33) is solved using the Matlab Robust Control Toolbox.

\section{Application to the Crazyflie quadrotor}

The previous results are now applied to the attraction domain estimation of P/PI controllers designed for the attitude control of a quadrotor. The following numerical values are choosen, corresponding to the model of our experimental platform Bitcraze Crazyflie 2.0:

$$
\begin{aligned}
J & =\operatorname{diag}\left(1.2 \times 10^{-5}, 1.2 \times 10^{-5}, 2 \times 10^{-5}\right) \\
D & =\operatorname{diag}\left(9.7 \times 10^{-6}, 9.7 \times 10^{-6}, 5.9 \times 10^{-5}\right)
\end{aligned}
$$

The gains of the controller are fixed to:

$$
\begin{aligned}
& k_{p_{\omega}}=\operatorname{diag}(250,250,50) \\
& k_{p_{\eta}}=\operatorname{diag}(32,32,32), k_{i_{\eta}}=\operatorname{diag}(10,10,10)
\end{aligned}
$$

The optimization problem (33) is solved numerically to compute the matrices $P=P^{\top}>0, W \in \mathcal{W}$ such that the asymptotic stability of the system is proven for the largest domain of initial conditions $\mathcal{E}_{p}$ contained in the largest set $B_{r}$ defined by the bounds on the angles $\phi$ and $\theta$. The maximum set $B_{r}$ was found to be $|\theta| \leq 30 \mathrm{deg}$ (resp. $\left.|\phi|\right)$ with the following $P$ matrix:

$P=\left[\begin{array}{ccccccccc}1.01 & 0 & 0 & 0.31 & 0 & 0 & 0.04 & 0 & 0 \\ 0 & 1.01 & 0 & 0 & 0.32 & 0 & 0 & 0.03 & 0 \\ 0 & 0 & 1.01 & 0 & 0 & 0.69 & 0 & 0 & 0.09 \\ 0.31 & 0 & 0 & 36.59 & 0 & 0 & 0.02 & 0 & 0 \\ 0 & 0.32 & 0 & 0 & 37.42 & 0 & 0 & 1.11 & 0 \\ 0 & 0 & 0.69 & 0 & 0 & 44.69 & 0 & 0 & 9.78 \\ 0.04 & 0 & 0 & 0.02 & 0 & 0 & 1.46 & 0 & 0 \\ 0 & 0.03 & 0 & 0 & 1.11 & 0 & 0 & 1.45 & 0 \\ 0 & 0 & 0.09 & 0 & 0 & 9.78 & 0 & 0 & 4.58\end{array}\right] \times 10^{-1}$

The figure 5 represents the set of bounds $B_{r}$ and the projection of the ellipsoid $\mathcal{E}_{p}$ onto the plan $(\phi, \theta)$. The projection of various trajectories of the nonlinear closed-loop system are plotted (dashed line). According to the previous study, for initial conditions taken in the ellipsoid $\mathcal{E}_{p}$, the trajectories of the nonlinear system remain in the bounded set $B_{r}$, as shown on figure 5 .

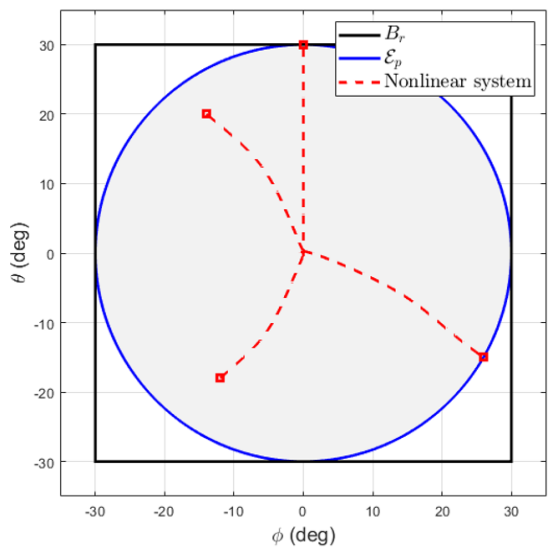

Fig. 5: Projection of $\mathcal{E}_{p}$ in the plan $(\phi, \theta)$

\section{CONCLUSION}

In this paper, the design of linear controllers for the nonlinear attitude model of the quadrotor has been investigated. Simple axis-decoupled P/PI controllers were designed using 
the linearisation of the nonlinear model at hover point and tuned using standard frequency design techniques. A method involving the resolution of LMI has been given to estimate the domain of attraction around the hover point of these controllers when they are applied to the nonlinear system. While generally corresponding to an infinite dimensional optimization problem, we showed that the specific structure of the VTOL attitude model such as the quadrotor one could be exploited to make this problem finite dimensional and thus numerically solvable. A numerical example showed that the P/PI controllers designed for our experimental quadrotor platform Bitcraze Crazyflie 2.0 stabilize the nonlinear model for roll and pitch angles up to $30 \mathrm{deg}$.

Finally, the present work provides a set of LMI conditions to evaluate the domain of attraction of a priori designed linear controllers for the VTOL nonlinear attitude model. These conditions could be extended in the future to perform the synthesis of nonlinear controllers as in [14] or to investigate the stability of the full VTOL nonlinear model including the translational motion.

\section{REFERENCES}

[1] R. Amin, L. Aijun, and S. Shamshirband, "A review of quadrotor UAV: Control methodologies and performance evaluation," International Journal of Automation and Control, vol. 10, no. 2, pp. 87-103, 2016.

[2] M. Hua, T. Hamel, P. Morin, and C. Samson, "Introduction to feedback control of underactuated VTOL vehicles: A review of basic control design ideas and principles," IEEE Control Systems Magazine, vol. 33, pp. 61-75, Feb. 2013.

[3] P. Bristeau, P. Martin, E. Salan, and N. Petit, "The role of propeller aerodynamics in the model of a quadrotor UAV," in 2009 European Control Conference (ECC), pp. 683-688, Aug. 2009.

[4] R. Fabela, C. Santana, A. Naranjo, L. Amezquita-Brooks, E. LiceagaCastro, and M. Torres-Reyna, "Experimental characterization of a small and micro unmanned aerial vehicle propulsion systems," in AIAA Atmospheric Flight Mechanics Conference, (San Diego, California, USA), American Institute of Aeronautics and Astronautics, Jan. 2016.

[5] A. L'Afflitto, R. B. Anderson, and K. Mohammadi, "An Introduction to Nonlinear Robust Control for Unmanned Quadrotor Aircraft: How to Design Control Algorithms for Quadrotors Using Sliding Mode Control and Adaptive Control Techniques [Focus on Education]," IEEE Control Systems Magazine, vol. 38, pp. 102-121, June 2018.

[6] T. Lee, M. Leok, and N. H. McClamroch, "Control of Complex Maneuvers for a Quadrotor UAV using Geometric Methods on SE(3)," arXiv:1003.2005 [cs, math], Mar. 2010. arXiv: 1003.2005.

[7] S. Bouabdallah, A. Noth, and R. Siegwart, "PID vs LQ control techniques applied to an indoor micro quadrotor," in 2004 IEEE/RSJ International Conference on Intelligent Robots and Systems (IROS) (IEEE Cat. No.04CH37566), vol. 3, (Sendai, Japan), pp. 2451-2456, IEEE, 2004.

[8] M. Dinh, G. Scorletti, V. Fromion, and E. Magarotto, "Parameter dependent $\mathrm{H}$-infinity control by finite dimensional LMI optimization: application to trade-off dependent control," International Journal of Robust and Nonlinear Control, vol. 15, no. 9, pp. 383-406, 2005.

[9] X. Zhang, X. Li, K. Wang, and Y. Lu, "A Survey of Modelling and Identification of Quadrotor Robot," Abstract and Applied Analysis, vol. 2014, 2014.

[10] J. P. How, B. Behihke, A. Frank, D. Dale, and J. Vian, "Real-time indoor autonomous vehicle test environment," IEEE Control Systems Magazine, vol. 28, pp. 51-64, Apr. 2008.

[11] F. Goodarzi, D. Lee, and T. Lee, "Geometric nonlinear PID control of a quadrotor UAV on SE(3)," in 2013 European Control Conference (ECC), (Zurich), pp. 3845-3850, IEEE, July 2013.

[12] S. Boyd, L. E. Ghaoui, E. Feron, and V. Balakrishnan, Linear Matrix Inequalities in System and Control Theory, vol. 15. SIAM, Jan. 1994.

[13] V. Fromion, G. Scorletti, and J. P. Barbot, "Quadratic observers for estimation and control in induction motors," in Proceedings of the 1999 American Control Conference, vol. 3, pp. 2143-2147 vol.3, June 1999.
[14] L. El Ghaoui and G. Scorletti, "Control of rational systems using linear-fractional representations and linear matrix inequalities," Automatica, vol. 32, pp. 1273-1284, Sept. 1996.

[15] G. H. Golub and C. F. Van Loan, Matrix computations. Johns Hopkins studies in the mathematical sciences, Baltimore: The Johns Hopkins University Press, fourth edition ed., 2013. OCLC: ocn824733531. 